\title{
ON EFFECTIVE IMPLICIT TIME INTEGRATION IN ANALYSIS OF FLUID-STRUCTURE PROBLEMS
}

\author{
KLAUS-JÜRGEN BATHE $\dagger$ AND VIJAY SONNAD $\ddagger$ \\ Department of Mechanical Engineering, Massachusetts Institute of Technology, Cambridge, Massachusetts, U.S.A.
}

\section{INTRODUCTION}

During recent years a considerable amount of research effort has been spent on the analysis of fluid-structure systems. An important feature of these analyses is in many cases the use of implicit-explicit time integration of the dynamic response: ${ }^{1,2}$ the response of the fluid is integrated using an explicit method (central difference technique) and the response of the structure is integrated with an implicit technique (e.g. the Newmark method). The principal advantage of such time integration of the dynamic response is that no stiffness matrix need be calculated for the fluid and a relatively large time step can be employed in the incremental analysis. Various schemes for performing the explicit-implicit integration have been proposed, and these were analysed for their stability and accuracy characteristics. ${ }^{1.3}$ These combined time integration techniques are not restricted in their use to the analysis of fluid-structure systems, but can be employed for the analysis of systems with 'stiff and flexible domains' in general.

The objective in this short paper is to point out that an effective solution of fluid-structure systems (and 'stiff and flexible domains' in general) can frequently also be calculated using an implicit time integration for the complete structural model with a lumped mass idealization and the recently proposed BFGS method. ${ }^{4.5}$ In the analysis procedure used, the response is calculated with implicit time integration for the fluid and the structure without setting up a stiffness matrix of the fluid, and by satisfying the dynamic equilibrium equations using the BFGS iterations.

\section{THE SOLUTION PROCEDURE}

The equations used in implicit time integration in a geometric and/or material nonlinear analysis are: ${ }^{5}$

$$
\mathbf{M}^{t+\Delta t} \ddot{\mathbf{U}}^{(i)}+{ }^{\tau} \mathbf{K} \Delta \mathbf{U}^{(i)}={ }^{t+\Delta t} \mathbf{R}-{ }^{t+\Delta t} \mathbf{F}^{(i-1)},
$$

where

$$
\begin{aligned}
\mathbf{M}= & \text { time independent mass matrix, } \\
{ }^{\tau} \mathbf{K}= & \text { tangent stiffness matrix corresponding to time } \tau, \\
{ }^{t+\Delta t} \mathbf{R}= & \text { vector of externally applied nodal loads corresponding to time } t+\Delta t, \\
{ }^{t+\Delta t}{ }^{(i-1)}= & \text { vector of nodal point forces equivalent to the element stresses at time } t+\Delta t \\
& \text { and iteration }(i-1), \\
{ }^{t+\Delta t} \ddot{\mathbf{U}}^{(i)}= & \text { vector of nodal point accelerations at time } t+\Delta t \text { and iteration }(i),
\end{aligned}
$$

$\dagger$ Associate Professor.

$\ddagger$ Graduate student.

0029-5981/80/0615-0943\$01.00

Received 29 October 1979

(C) 1980 by John Wiley \& Sons, Ltd. 
and

$$
{ }^{t+\Delta t} \mathbf{U}^{(i)}={ }^{\imath+\Delta t} \mathbf{U}^{(i-1)}+\Delta \mathbf{U}^{(i)},
$$

where $\Delta \mathbf{U}^{(i)}$ is the incremental nodal point displacement vector corresponding to time $t$ and iteration $(i)$.

Using the trapezoidal rule of time integration, the following assumptions are employed:

$$
\begin{aligned}
& { }^{t+\Delta t} \mathbf{U}={ }^{t} \mathbf{U}+\frac{\Delta t}{2}\left({ }^{t} \dot{\mathbf{U}}+{ }^{t+\Delta t} \dot{\mathbf{U}}\right) \\
& { }^{t+\Delta t} \dot{\mathbf{U}}={ }^{t} \dot{\mathbf{U}}+\frac{\Delta t}{2}\left({ }^{t} \ddot{\mathbf{U}}+{ }^{t+\Delta t} \ddot{\mathbf{U}}\right) .
\end{aligned}
$$

Using equations (2)-(4) we obtain

$$
{ }^{t+\Delta t} \ddot{\mathbf{U}}^{(i)}=\frac{4}{\Delta t^{2}}\left({ }^{t+\Delta t} \mathbf{U}^{(i-1)}+\Delta \mathbf{U}^{(i)}-{ }^{t} \mathbf{U}\right)-\frac{4}{\Delta t}{ }^{t} \dot{\mathbf{U}}-{ }^{t} \ddot{\mathbf{U}}
$$

and substituting into equation (1) we have

$$
{ }^{\tau} \hat{\mathbf{K}} \Delta \mathbf{U}^{(i)}={ }^{t+\Delta t} \mathbf{R}-{ }^{t+\Delta t} \mathbf{F}^{(i-1)}-\mathbf{M}\left[\frac{4}{\Delta t^{2}}\left({ }^{t+\Delta t} \mathbf{U}^{(i-1)}-{ }^{t} \mathbf{U}\right)-\frac{4}{\Delta t} \dot{\mathbf{U}}-{ }^{t} \dot{\mathbf{U}}\right]
$$

where

$$
{ }^{\tau} \hat{\mathbf{K}}={ }^{\tau} \mathbf{K}+\frac{4}{(\Delta \mathrm{t})^{2}} \mathbf{M}
$$

In the BFGS iteration, equation (6) is solved as follows:

$$
\Delta \mathbf{U}^{(i)}=\left({ }^{\tau} \hat{\mathbf{K}}^{-1}\right)^{(i-1)}\left({ }^{t+\Delta t} \mathbf{R}-\mathbf{M}^{t+\Delta t} \ddot{\mathbf{U}}^{(i-1)}-{ }^{t+\Delta t} \mathbf{F}^{(i-1)}\right)
$$

and

$$
{ }^{t+\Delta t} \mathbf{U}^{(i)}={ }^{t+\Delta t} \mathbf{U}^{(i-1)}+\beta \Delta \mathbf{U}^{(i)},
$$

where $\beta$ is a scalar that is evaluated by a line search to satisfy the condition

$$
\Delta \mathbf{U}^{(i) \mathrm{T}}\left({ }^{t+\Delta t} \mathbf{R}-{ }^{t+\Delta t} \mathbf{F}^{(i)}-\mathbf{M}^{t+\Delta t} \ddot{\mathbf{U}}^{(i)}\right) \leqslant \operatorname{STOL} \Delta \mathbf{U}^{(i) \mathrm{T}}\left({ }^{t+\Delta t} \mathbf{R}-\mathbf{M}^{t+\Delta t} \ddot{\mathbf{U}}^{(i-1)}-{ }^{t+\Delta t} \mathbf{F}^{(i-1)}\right)
$$

with STOL a tolerance. The coefficient matrix in equation (8) is evaluated as follows:

$$
\left({ }^{\tau} \hat{\mathbf{K}}^{-1}\right)^{(i-1)}=\mathbf{A}^{(i-1) \mathrm{T}}\left({ }^{\tau} \hat{\mathbf{K}}^{-1}\right)^{(i-2)} \mathbf{A}^{(i-1)} ; \quad{ }^{\top} \hat{\mathbf{K}}^{(0)}={ }^{\tau} \hat{\mathbf{K}},
$$

where the matrices $\mathbf{A}^{(i-1)}$ are of the simple form $\mathbf{A}^{(i-1)}=I+v^{(i-1)} \mathbf{w}^{(i-1) \mathrm{T}}$. The vectors $\mathbf{w}^{(i-1)}$ and $v^{(i-1)}$ are given by the calculated nodal point displacements and forces. Convergence is achieved in the iteration when the following two criteria are satisfied:

$$
\frac{\left\|^{t+\Delta t} \mathbf{R}-{ }^{t+\Delta t} \mathbf{F}^{(i-1)}-\mathbf{M}^{t+\Delta t} \ddot{\mathbf{U}}^{(i-1)}\right\|_{2}}{\left\|{ }^{k} \mathbf{R}-{ }^{\kappa-\Delta t} \mathbf{F}-\mathbf{M}^{\kappa-\Delta t} \ddot{\mathbf{U}}\right\|_{2}^{(\max )}} \leqslant \text { RTOL, }
$$

where $\kappa$ is any one of the discrete times considered, and

$$
\frac{\Delta \mathbf{U}^{(i) \mathrm{T}}\left({ }^{t+\Delta t} \mathbf{R}-{ }^{t+\Delta t} \mathbf{F}^{(i-1)}-\mathbf{M}^{t+\Delta t} \ddot{\mathbf{U}}^{(i-1)}\right)}{\Delta \mathbf{U}^{(1) \mathrm{T}}\left({ }^{t+\Delta t} \mathbf{R}-{ }^{t} \mathbf{F}-\mathbf{M}^{t} \ddot{\mathbf{U}}\right)} \leqslant \text { ETOL, }
$$


where RTOL is an out-of-balance force tolerance and ETOL is an 'energy' tolerance. In equation (12) the superscript (max) denotes the maximum value ever calculated during the solution, and $\|\mathbf{a}\|_{2}$ is the Euclidean norm of the vector $\mathbf{a}^{6}$

The above equations have been written for nonlinear dynamic analysis, but they are equally applicable in linear dynamic analysis and static analysis (in which case the inertia forces must simply be neglected).

An important point to note is that in the iteration of equations (8)-(11), a very approximate stiffness matrix can be used, and indeed such use may yield a most effective solution if the number of iterations required for convergence is not excessive. For example, it may be effective to use throughout a nonlinear incremental static analysis the initial elastic stiffness matrix, in which case only one stiffness matrix calculation and $\mathbf{L D L}{ }^{\mathrm{T}}$ decomposition at the beginning of the solution is required. However, the number of iterations required to solve the equilibrium equations increases when the matrix ${ }^{\tau} \mathbf{K}$ approximates the actual tangent stiffness matrix of the system less accurately.

Considering a dynamic analysis of a fluid-structure system, it is recognized that as the time step $\Delta t$ becomes small, the contribution of the mass matrix to the effective stiffness matrix becomes predominant in the fluid domain. If in such analysis a lumped mass matrix is used, it can be effective not to include the fluid element stiffness matrix contributions to the matrix ${ }^{\top} \hat{\mathbf{K}}$. This is particularly the case when the fluid domain is large (modelled using a large number of elements) compared to the structure. Thus, if we write,

$$
{ }^{\top} \mathbf{K}={ }^{\top} \mathbf{K}_{\mathbf{s}}+{ }^{\top} \mathbf{K}_{\mathbf{f}},
$$

where ${ }^{\top} \mathbf{K}_{\mathrm{s}}$ and ${ }^{\top} \mathbf{K}_{\mathrm{f}}$ are the stiffness matrices of the structure and the fluid, respectively, it can be effective to use in equation (11)

$$
{ }^{\top} \hat{\mathbf{K}}^{(0)}={ }^{\top} \mathbf{K}_{\mathrm{s}}+\frac{4}{\Delta t^{2}} \mathbf{M}
$$

The use of equation (15) may decrease the bandwidth of the coefficient matrix a great deal. Considering the computations, the solution scheme is now similar to an explicit time integration of the fluid response and an implicit integration of the structural response, but in fact, by the iteration, all finite element equations are integrated implicitly.

The procedure is appealing because the integration scheme does not require the use of special interface conditions, and the method is not subject to severe time step size constraints in the fluid domain.

\section{SAMPLE ANALYSIS}

The BFGS iteration scheme is available in the ADINA computer program, ${ }^{7}$ which was modified for this analysis not to include the stiffness contributions of the fluid elements in the coefficient matrix.

The fluid-structure problem considered is shown in Figure 1. A similar problem was already earlier analysed by Belytschko and Mullen using explicit-implicit integration. ${ }^{1}$ Figure 2 shows the $x$-velocities predicted at points $A$ and $B$ as a function of time. It should be noted that our objective was only to study the use of the proposed technique for the solution of the finite element equilibrium equations of this model and not to investigate whether this model does represent the physical situation in an accurate manner.

In the first analysis, the response was calculated using the usual solution procedure with the implicit trapezoidal rule and $\Delta t=10^{-5} \mathrm{sec}^{6}$ Next, the response was calculated with the BFGS 


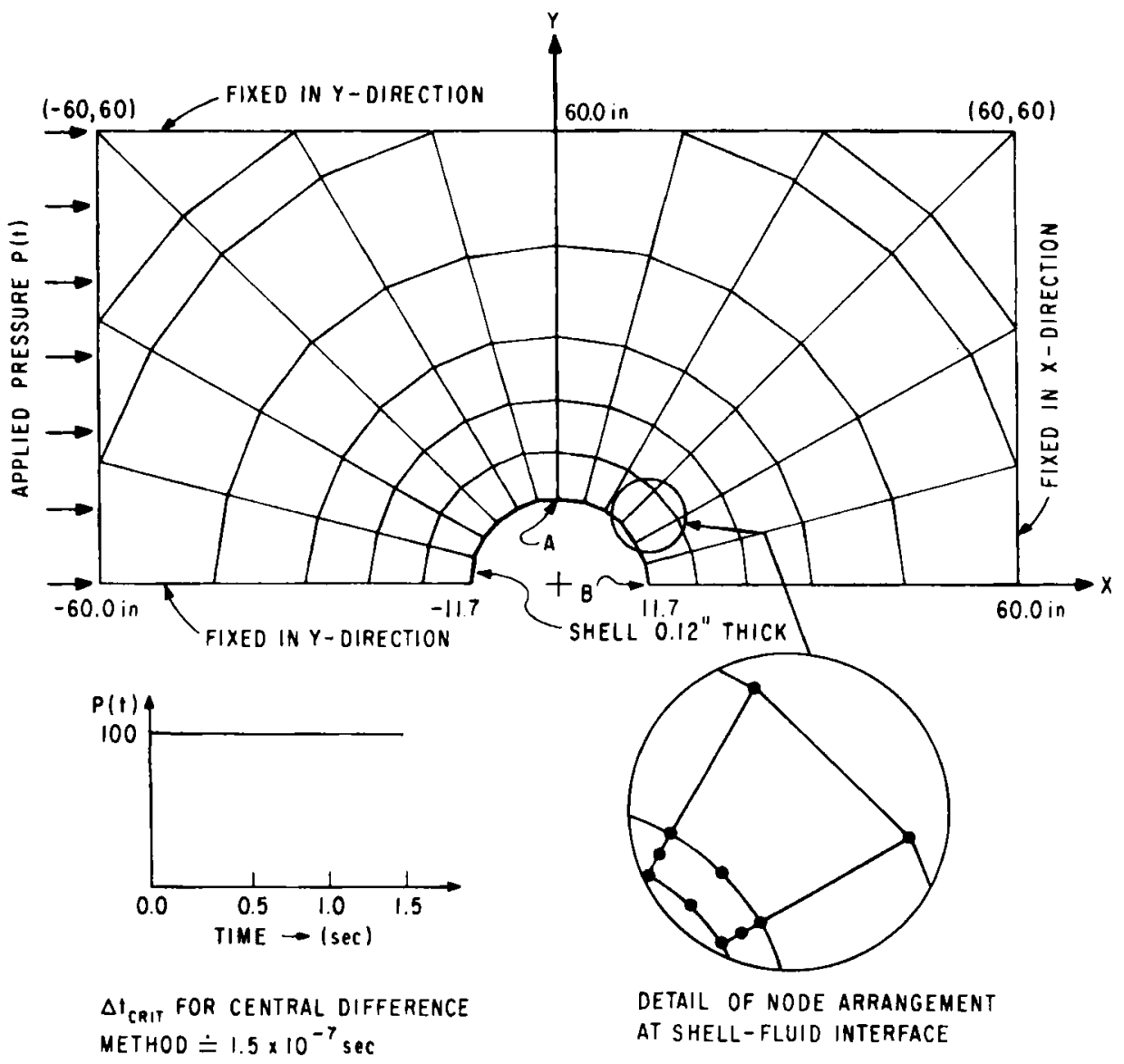

MATERIAL PROPERTIES (plone stroin model)

$\begin{array}{ll}\text { BULK MODULUS OF FLUID } & =3.0 \times 10^{5} \mathrm{psi} \\ \text { DENSITY OF FLUID } & =9.35 \times 10^{-5} \mathrm{lbs} / \mathrm{in}^{4}-\mathrm{sec}^{2} \\ \text { YOUNG'S MODULUS OF SHELL } & =3.0 \times 10^{7} \mathrm{pSi} \\ \text { POISSON'S RATIO OF SHELL } & =0.25 \\ \text { DENSITY OF SHELL } & =7.35 \times 10^{-4} \mathrm{lbs} / \mathrm{in}^{4}-\mathrm{sec}^{2}\end{array}$

Figure 1. Analysis of long cylindrical shell surrounded by fluid; fluid is subjected to step pressure

method with the starting coefficient matrix of equation (15) and using $\Delta t=10^{-5}$ and $10^{-4} \mathrm{sec}$. These two analyses were also performed using the modified Newton method with the constant coefficient matrix equal to the matrix in equation (15), in order to evaluate its effectiveness as well. Table I shows the average and maximum number of iterations required per time step in these computations. Figure 2 shows that, for the smaller time step, practically identical responses are predicted using any one of the procedures. But Table I shows that, for the larger time step, the Newton method failed to converge within 30 iterations at the eighth time step, whereas the BFGS method never required more than 5 iterations per time step. In all the above analyses we used equations (12) and (13) with $\mathrm{RTOL}=10^{-3}$ and $E T O L=10^{-5}$. 

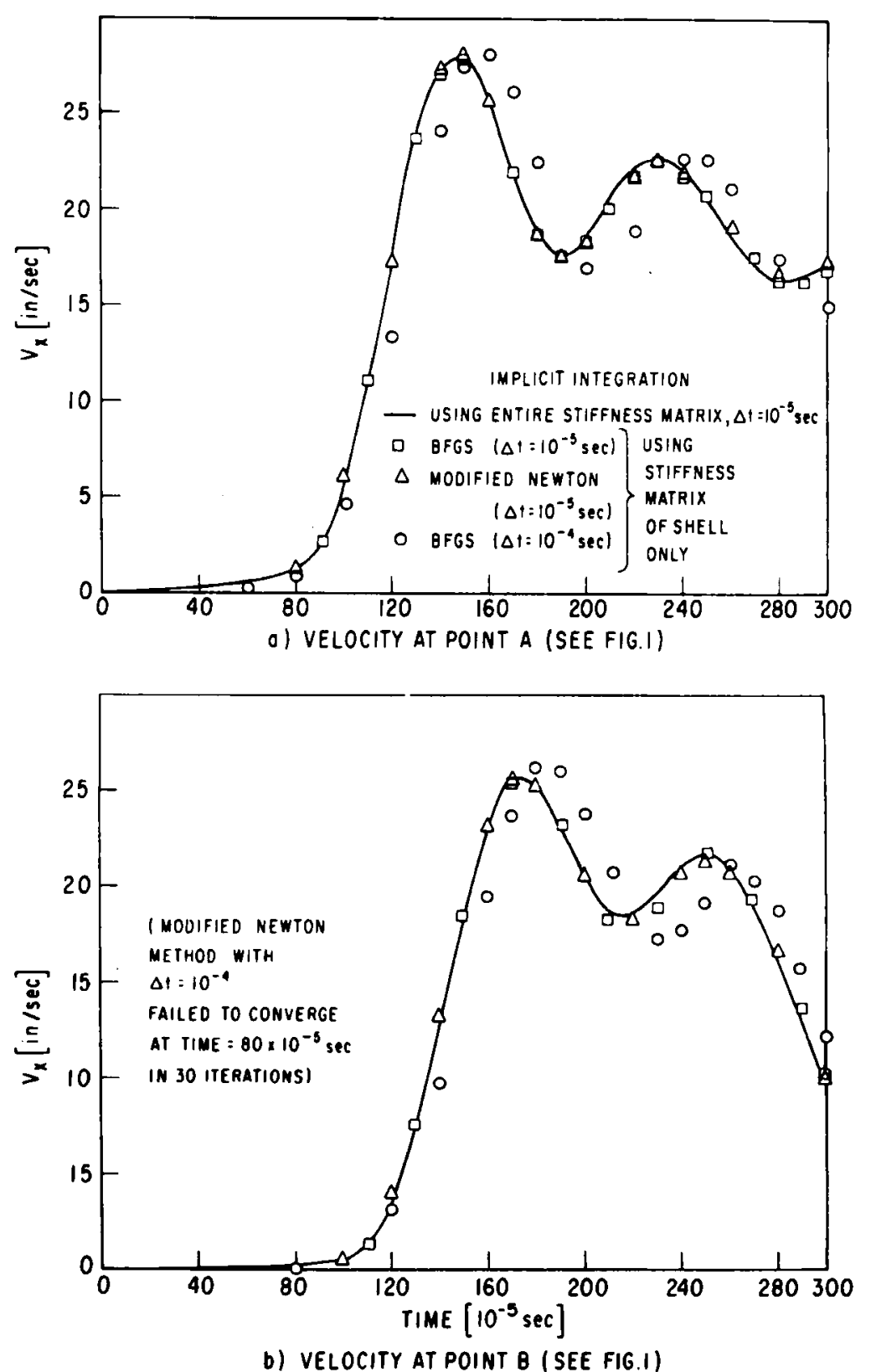

Figure 2. Velocity response at points $A$ and $B$ of the shell

\section{CONCLUSIONS}

An effective application of the BFGS method to some wave propagation fluid-structure problems was pointed out. The essence of the solution is that the stiffness matrix contributions of the fluid elements are neglected in the starting coefficient matrix of the iteration. It is concluded that in nonlinear dynamic analysis, in which iteration is required anyway in order to preserve the 
Table I. Number of iterations used in solutions

\begin{tabular}{lcccc}
\hline & $\Delta t=10^{-5} \mathrm{sec}$ & & \multicolumn{2}{c}{$\Delta t=10^{-4} \mathrm{sec}$} \\
\hline BFGS & Maximum & Average & Maximum & Average \\
Modified Newton & 2 & 2 & 5 & 4 \\
& 2 & 2 & $\begin{array}{l}\text { Failed to converge in } \\
\text { 30 iterations }\end{array}$ \\
\hline
\end{tabular}

stability of the solution ${ }^{8}$ and the fluid element stiffness effects in the coefficient matrix are small, this iterative technique may be very efficient. The solution scheme has the computational advantage of explicit-implicit time integration that no coefficient matrix corresponding to the fluid elements needs to be assembled and dealt with, and has as an additional advantage the stability of the implicit time integration in the fluid domain. The method can be cost-effective as a practical tool because of the good convergence characteristics of the BFGS method.

\section{REFERENCES}

1. T. Belytschko and R. Mullen, 'Mesh partitions of explicit-implicit time integration', in Formulations and Computational Algorithms in Finite Element Analysis (Eds. K. J. Bathe, J. T. Oden and W. Wunderlich), M.I.T. Press, 1977.

2. T. Belytschko, H. J. Yen and R. Mullen, 'Mixed methods for time integration', J. Comp. Meth. Appl. Mech. Engng, 17/18, 259-275 (1979).

3. T. J. R. Hughes and W. K. Liu, 'Implicit-Explicit finite elements in transient analysis: stability theory' J. Appl. Mech. 45 (June 1978).

4. H. Matthies and G. Strang, 'The solution of nonlinear finite element equations', Int. J. num. Meth. Engng, in press.

5. K. J. Bathe and A. P. Cimento, 'Some practical procedures for the solution of nonlinear finite element equations', J. Comp. Meth. Appl. Mech. Engng, in press.

6. K. J. Bathe and E. L. Wilson, Numerical Methods in Finite Element Analysis, Prentice-Hall, Englewood Cliffs, N.J., 1976.

7. K. J. Bathe, 'ADINA-a finite element program for automatic dynamic incremental nonlinear analysis', Report A VL 82448-1, Department of Mechanical Engineering, M.I.T., 1975 (rev. Dec. 1978).

8. K. J. Bathe, 'Finite element formulation, modeling and solution of nonlinear dynamic problems', in Numerical Methods for Partial Differential Equations, (Ed. S. V. Parker), Academic Press, London and New York, 1979. 